\title{
Editorial Statement
}

1996 was an unusual year for Computing Surveys, with two special issues: one devoted to perspectives in computing (March) and the other to strategic directions in computing (December). We would like to hear from readers about these issues, and more generally about whether the new look that mixes traditional papers with symposia of short papers on special topics serves the readership (please send mail to csur@acm.org). The present issue returns to a traditional form with three papers on image coding, nondeterminism, and cache coherence.

Reid, Millar, and Black in their paper distinguish second-generation image coding, which makes use of geometrical features of images, from first-generation coding based on pixel representation. It examines image sensitivity of the human visual system and techniques like multiscale edge detection and digital filtering that take this semantics into account. Visual pattern-based approaches using quadtrees are contrasted with segmentation-based approaches using techniques such as polynomial approximation, region-growing, split and merge techniques, and fractal coding techniques. Contour-coding-based approaches with lossy coding and lossless compression are examined. The conclusion views image coding as a two-stage modeling and coding process and reexamines previously discussed techniques in terms of their modeling and coding strategies. The mix between discussion of high-level modeling strategies and low-level algorithms gives readers an understanding of both strategies and tactics for second-generation image coding.

The paper by Walicki and Meldal breaks new ground in its comprehensive analysis of nondeterminism. It distinguishes between syntactic nondeterminacy of alternative ways of realizing a deterministic result, nondeterminism due to underspecification of processes, semantic nondeterminism that yields genuine nondeterminism, and arbitrary choice among alternatives. Varieties of nondeterminism considered include singular versus plural parameter-passing as well as weak versus strong, tight versus loose, and restrained versus unrestrained nondeterminism. Algebraic nondeterminism is modeled by parameters or multialgebras whose values are sets of nondeterministic elements. The body of this paper is devoted to lattice-theoretic results for algebras and relational systems that only accomplished mathematical readers can follow. However, the introduction and the concluding sections provide intuitions concerning nondeterminism accessible to the nonspecialist reader.

Pong and Dubois examine verification techniques for cache coherence-the problem of maintaining consistency of multiple copies of cached data. It applies model-checking and symbolic state models to the analysis of memory architectures, including uniform memory access (UMA), nonuniform memory access (NUMA), and cache-only memory access (COMA) models. Cache-coherence protocols, memory-consistency models, and latency-tolerance techniques are presented to provide a concrete framework for finite-state verification models. Protocols are modeled at the behavior level assuming atomic protocol transactions, at the message-passing or system levels assuming nonatomic protocols, and at the architectural level considering implementation details. A multiprocessor system with coherent caches modeled by single memory locations is used as a running example. Model-checking based on computational tree logic (CTL) is used as a framework for verification. One of this paper's strengths is its detailed presentation of both an application domain and model-checking techniques for the analysis of application domains, 
providing a bridge between theory and practice. Systematic model checking and symbolic state models for controlling the state-space explosion are explored. Alternative verification methods based on state enumeration, symbolic model checking, and symbolic state models are evaluated and compared. This article illustrates both the growing maturity of model-checking methods and practical limitations of model-checking arising from the inability to control state explosion.

In conjunction with ACM student chapters, Computing Surveys is launching a student paper contest to encourage expository writing among undergraduate and graduate students. We hope there will be strong grassroots support for this initiative among chapters, with local student contests coordinated with classes having term paper projects. Submissions will be judged by a panel of students who will present their recommendations to a Computing Surveys editorial panel. The best paper will be published in Surveys, while other deserving papers will be published in the student journal Crossroads. Further information can be obtained from John Cavazos, student coordinator of the tutorial paper contest as well as a editor of Crossroads (cavazos@cs.umass.edu).

Finally, we look ahead to the year 2047, when the ACM and the discipline of computing will be 100 years old. At ACM '97, the question of whether 2047 was a prime number was a topic of discussion among forward-looking planners. It turns out that 2047 is a Mersenne number, and Knuth's analysis of Mersenne numbers in The Art of Computer Programming shows that Mersenne numbers $2 * * p-1$ are prime unless they have factors of the form $2 k p+1$ for some integer $k$. The number 2047 has no obvious factors, and planners were proceeding on the hypothesis that 2047 was prime when it was discovered that 2047 is in fact $23 * 89$ (both factors have the form $22 k+1$ as required). This was discovered just as the press was about to announce that 2047 would be a prime year for computing. We hope that this knowledge will be useful in long-term planning for the ACM.

Peter Wegner

Editor-in-Chief

\section{ACKNOWLEDGMENT}

We gratefully acknowledge support by the SIG discretionary fund of the December Strategic Directions issue of Computing Surveys, and apologize for our failure to include an acknowledgment in the December issue itself. 